\title{
La résistance aux benzimidazoles des nématodes gastro-intestinaux des petits ruminants en Gambie et au Sénégal
}

\author{
H. Bâ ${ }^{1,2}$ S. Geerts ${ }^{1 *}$
}

Mots-clés

O vin - Caprin - Nematoda - Benzimidazole - Résistance aux produits chimiques - Anthelminthique - Gambie Sénégal.

\begin{abstract}
Résumé
Cinquante-cinq troupeaux de petits ruminants en Gambie et au Sénégal ont été examinés en utilisant le test d'inhibition de l'éclosion des œufs et/ou le test de réduction des œufs de nématodes dans les fèces afin de détecter une résistance éventuelle aux benzimidazoles. Sur 34 troupeaux villageois examinés, quatre cas de suspicion de résistance aux benzimidazoles ont été identifiés. En revanche, chez 21 troupeaux améliorés deux cas de résistance et quatre cas de suspicion de résistance ont été constatés. Cette étude a permis de mettre en évidence pour la première fois des cas de résistance aux benzimidazoles chez les nématodes gastro-intestinaux des petits ruminants au Sénégal et en Gambie. Des recherches devraient être faites sur une plus grande échelle afin de mesurer la prévalence de la résistance des nématodes aux anthelminthiques dans la région et de déterminer les espèces de parasites mises en cause.
\end{abstract}

\section{INTRODUCTION}

Le développement de la résistance aux benzimidazoles chez les nématodes gastro-intestinaux est devenu un problème crucial dans les élevages de petits ruminants à travers le monde $(6,9,15)$. De plus en plus de cas de chimiorésistances multiples aux benzimidazoles, au lévamisole, aux salicylanilides et aux ivermectines sont signalés chez les espèces Haemonchus contortus et Trichostrongylus colubriformis dans les pays tropicaux $(2,9,14)$. Jusqu'à présent aucun cas de résistance aux anthelminthiques n'a été décrit en Afrique occidentale.

Au Sénégal et en Gambie, où le parasitisme intestinal est l'obstacle majeur au développement de l'élevage et où $H$. contortus et T. colubriformis sont les vers les plus répandus dans ces régions $(8,12)$, le problème mérite d'être étudié. L'objectif de la présente étude était de vérifier s'il y avait de la résistance aux benzimidazoles chez les nématodes gastro-intestinaux des petits ruminants d'élevages villageois et d'élevages (semi-)améliorés dans certaines régions de la Gambie et du Sénégal.

1. Institut de médecine tropicale Prince Léopold, Département vétérinaire, 155 Nationalestraat, B-2000 Antwerpen 1, Belgique

2. Adresse actuelle : E 78 Patte d'Oie Builders, Dakar, Sénégal

* Auteur pour la correspondance

Tel : +32 32476262 ; Fax : +3232476268

E-mail : sgeerts@itg.be

\section{MATERIEL ET METHODES}

\section{Zone d'étude}

Le travail a été réalisé pendant la saison des pluies d'août 1995 à janvier 1996 et de juillet 1996 à octobre 1996. En Gambie les élevages se situaient dans trois régions différentes qui s'étendent sur une longueur de $315 \mathrm{~km}$ le long du fleuve Gambie (Banjul, Kenaba et Bansang). Au Sénégal, la région de Kolda et la zone des Niayes (comprises entre Dakar et Thies) ont été concernées par cette étude. Le climat est de type soudano-sahélien à Kolda tandis que les Niayes sont situées sur une importante nappe phréatique favorable à l'élevage intensif du bétail et des volailles.

\section{Choix des troupeaux et des anthelminthiques}

Le choix des troupeaux n'a pas été fait complètement au hasard, mais selon la disponibilité des éleveurs et l'accessibilité des élevages. Cependant, dans la sélection des troupeaux il n'a pas été tenu compte d'indications de problèmes éventuels de résistance aux anthelminthiques. L'enquête a porté sur un total de 55 troupeaux de petits ruminants, dont 34 troupeaux villageois où le traitement aux anthelminthiques se faisait de façon sporadique et où les animaux ne recevaient aucun soin particulier, et 21 troupeaux améliorés où deux traitements aux anthelminthiques au moins étaient administrés par an et où les animaux étaient complémentés. L'albendazole (suspension orale 2,5 p. 100, Phénix, Belgique) ou le fenbendazole (Panacur, Hoechst, suspension orale 2,5 p. 100) à la dose de $5 \mathrm{mg} / \mathrm{kg}$ recommandée par le fabricant ont été testés dans ces troupeaux. 


\section{Prélèvements des échantillons de matières fécales}

Dans chaque troupeau 10 à 20 échantillons de matières fécales ont été prélevés par voie rectale. Approximativement 3 à $5 \mathrm{~g}$ de matières fécales de différents échantillons ont été mélangés et broyés afin de préparer un mélange (15 g) qui a été conservé en anaérobiose selon la méthode de Hunt et Taylor (10) pour le test d'inhibition de l'éclosion des œufs. Dans les troupeaux, où le test de réduction des œufs dans les matières fécales a été fait, les échantillons de matières fécales étaient pris séparément et conservés dans des sachets en plastique identifiés et acheminés au laboratoire dans les 2 à $24 \mathrm{~h}$ après les prélèvements et conservés aux frais jusqu'à l'examen.

\section{Test d'inhibition de l'éclosion des aufs (egg hatch test)}

Ce test a été utilisé dans 38 élevages (31 améliorés et 7 villageois), dont 13 en Gambie et 25 au Sénégal. Dans quelques élevages, le test d'inhibition de l'éclosion des œufs a été fait en parallèle avec le test de réduction du nombre d'œufs par gramme de fèces (OPG). La méthode de Le Jambre (11) modifiée par Coles et coll. (5) a été utilisée pour détecter la résistance. Les matières fécales prises avant le traitement ont été conservées en anaérobiose et analysées dans un délai de 1 à 5 jours après la collecte.

Au total, cinq concentrations de thiabendazole ont été testées, soit respectivement $2,0,5,0,1,0,05$ et $0,025 \mu \mathrm{g} / \mathrm{ml}$. Chaque concentration a été répétée au moins deux fois et, pour la plupart, trois fois. La DL50 (dose létale qui prévient 50 p. 100 des œufs d'éclore) a été calculée selon la méthode du probit. Une DL50 entre 0,10 et $0,11 \mu \mathrm{g} / \mathrm{ml}$ est considérée comme une suspicion de résistance, tandis qu'une DL50 de $0,12 \mu \mathrm{g} / \mathrm{ml}$ est indicative de vraie résistance (4).

\section{Test de réduction des oufs dans les matières fécales}

Ce test a été fait dans 23 troupeaux (11 en Gambie et 12 au Sénégal), dont 16 troupeaux améliorés et 7 villageois. Dans chaque troupeau les animaux ont été identifiés avec les boucles auriculaires puis répartis en deux groupes : 11 animaux en moyenne pour le groupe traité et 8 animaux en moyenne pour le groupe témoin. Tous les animaux d'un troupeau ont reçu une dose d'anthelminthique égale à celle de l'animal le plus lourd du troupeau qui a été préalablement pesé.
L'OPG avant traitement et les OPG entre 10 et 14 jours après traitement ont été évalués avec la méthode McMaster modifiée, décrite par Coles et coll. (5).

Le pourcentage de réduction des OPG a été calculé en utilisant la méthode de la World Association for the Advancement of Veterinary Parasitology (WAAVP) (5). On considère qu'il y a de la résistance, d'une part, si le pourcentage de réduction de l'OPG est inférieur à 95 p. 100 et, d'autre part, si la limite inférieure de l'intervalle de confiance (95 p. 100) est en dessous de 90 p. 100. La résistance est suspectée si seulement un des deux critères est rempli.

Le test de réduction des œufs dans les matières fécales n'a pas pu être fait dans tous les élevages visités du fait que de nombreux troupeaux étaient suivis par l'un ou par l'autre projet de recherche (sous la supervision du Centre international de trypanotolérance, Banjul, ou du Centre de recherche zootechnique de Kolda). Lorsque c'était le cas, le test d'inhibition de l'éclosion des œufs a été effectué.

\section{Questionnaire}

Un questionnaire a été distribué à chaque propriétaire de troupeau pour recueillir les informations relatives aux techniques de production adoptées, aux différentes espèces animales élevées, à l'utilisation d'anthelminthiques et aux contraintes liées à l'utilisation des anthelminthiques dans l'élevage.

\section{RESU LTATS}

\section{Troupeaux villageois}

Sur 15 troupeaux examinés (neuf ovins et six caprins), deux cas de suspicion de résistance à l'albendazole ou au fenbendazole (un troupeau ovin et un caprin) ont été trouvés en Gambie en utilisant le test de réduction de l'OPG. Le test d'inhibition de l'éclosion des œufs a révélé deux cas de suspicion de résistance aux benzimidazoles dans 11 troupeaux caprins villageois examinés à Kolda (tableau I). Chez les huit troupeaux ovins (Sénégal), aucun cas de résistance n'a été détecté en utilisant le test d'inhibition de l'éclosion des œufs.

Tableau I

Troupeaux villageois : résultats du test de réduction des œufs dans les matières fécales et du test d'inhibition de l'éclosion des œufs

\begin{tabular}{|c|c|c|c|c|c|c|}
\hline Zone d'étude & Nb. testés & Nb. R ou SU SP & Localité & $\begin{array}{c}R \\
(\%)\end{array}$ & $\begin{array}{l}\text { LIC } \\
(\%)\end{array}$ & $\begin{array}{c}\text { D L50 TBZ } \\
(\mu \mathrm{g} / \mathrm{ml})\end{array}$ \\
\hline \multicolumn{7}{|l|}{ G ambie } \\
\hline - O vins & 9 & 1 SU SP & Bansang & 95,4 & 75,8 & $<0,05$ \\
\hline - Caprins & 6 & 1 SU SP & Kenaba & 98 & 82,2 & - \\
\hline \multicolumn{7}{|l|}{ Sénégal } \\
\hline - Ovins & 8 & 0 & Kolda & NT & - & 0,02 à 0,08 \\
\hline - Caprins & 11 & $\begin{array}{l}1 \text { SU SP } \\
1 \text { SU SP }\end{array}$ & $\begin{array}{l}\text { Kolda } \\
\text { Kolda }\end{array}$ & $\begin{array}{l}\text { NT } \\
\text { NT }\end{array}$ & $\begin{array}{l}- \\
-\end{array}$ & $\begin{array}{c}0,1 \\
0,11\end{array}$ \\
\hline
\end{tabular}

$\mathrm{R}(\%)=$ pourcentage de réduction $($ WAAVP) $;$ LIC $=$ limite inférieure $(95 \%)$ de confiance $; \mathrm{NT}=$ non testé $;$ DL50 = dose létale qui inhibe l'éclosion de $50 \%$ des cufs incubés $; \mathrm{TBZ}=$ thiabendazole $; \mathrm{Nb}$. testés $=$ nombre de troupeaux testés $; \mathrm{R}=$ résistance $; \mathrm{SUSP}=$ suspicion de résistance 


\section{Troupeaux améliorés}

Dans les quatre élevages de petits ruminants (trois ovins et un caprin) en Gambie, deux cas de résistance à l'albendazole chez les ovins et un cas de suspicion de résistance au fenbendazole chez les caprins ont été trouvés avec la méthode de Coles et coll. (5). Dans un des troupeaux ovins, où le test d'inhibition de l'éclosion des œufs a été fait en parallèle, la résistance n'a pas pu être confirmée par le dernier test. Au Sénégal, trois cas de suspicion de résistance ont été détectés dans 15 élevages ovins améliorés. Parmi les deux élevages caprins, aucun cas de résistance n'a été observé (tableau II).

Il n'y avait pas de différence significative entre le nombre de cas de résistance $(\mathrm{p}=0,16)$ et de suspicion de résistance $(\mathrm{p}=0,17)$ dans les élevages améliorés (respectivement $2 / 21$ et $4 / 21$ ) et dans les élevages villageois (respectivement 0/34 et 4/34).

\section{Résultats du questionnaire}

Les résultats de l'enquête indiquent que 70,5 p. 100 des propriétaires de troupeaux villageois ont eu recours à des traitements traditionnels de déparasitage avec l'écorce de certains arbres, tels que le Caecedra, contre 14,3 p. 100 dans les troupeaux améliorés. Les informations recueillies auprès des agents du Centre international de trypanotolérance responsables du suivi de certains troupeaux villageois en Gambie et des agents du Centre de recherche zootechnique de Kolda indiquent que les traitements au fenbendazole ont été appliqués sporadiquement dans le cadre de certains projets de recherche dans les villages concernés par cette étude. Dans les troupeaux améliorés au moins deux traitements par an étaient administrés. L'ivermectine, le fenbendazole et le pyrantel étaient utilisés en ordre décroissant.

\section{DISCUSSION}

\section{Troupeaux villageois}

Il était assez surprenant que des cas de suspicion de résistance (4/34 ou 11,8 p. 100) aient été détectés dans les troupeaux villageois, car il n'existait aucun programme de traitement contre les vers gastro-intestinaux dans ces élevages. Des enquêtes menées auprès des éleveurs ont montré que la plupart des troupeaux étaient sous le suivi de certains projets de recherche. Dans le cadre de ces projets, des anthelminthiques ont été administrés sporadiquement, ce qui pourrait expliquer le taux de suspicion de résistance observé.

\section{Troupeaux améliorés}

Les deux cas de résistance aux benzimidazoles qui ont été observés étaient des élevages sous le suivi intensif d'un centre de recherche. Bien qu'au cours des dernières années le nombre de traitements par an n'y ait pas été exceptionnellement élevé (environ deux par an), cela a suffi pour causer des problèmes de résistance $(1,7)$.

Dans ce travail il y a une corrélation très faible entre le test de réduction des œufs et le test d'inhibition de l'éclosion. Ce phénomène a aussi été observé par Dorny et coll.(7) en Malaisie où de nombreux cas de résistance détectés en utilisant le test de réduction des œufs n'étaient pas confirmés par le test in vitro d'inhibition de l'éclosion.

Pour ce qui concerne les résultats des tests de réduction des œufs chez les caprins, il faut les interpréter avec réserve, parce que la dose utilisée $(5 \mathrm{mg} / \mathrm{kg})$, qui est toujours recommandée par le fabricant, est en fait insuffisante et devrait être doublée en raison de la biodisponibilité plus faible des benzimidazoles chez les chèvres $(3,13)$.

\section{CONCLUSION}

Cette étude a mis en évidence pour la première fois des cas de résistance aux benzimidazoles chez les nématodes gastro-intestinaux de moutons en Afrique occidentale. Les espèces d'helminthes résistants n'ont pas été identifiées et elles pourraient constituer le projet d'un travail ultérieur. Des recherches sur une échelle plus grande devraient être faites afin de mesurer la prévalence de la résistance anthelminthique dans la région.

\section{Tableau II}

Troupeaux améliorés : résultats du test de réduction des œufs dans les matières fécales et du test d'inhibition de l'éclosion des œufs

\begin{tabular}{|c|c|c|c|c|c|c|}
\hline Zone d'étude & N b. testés & Nb. R ou SU SP & Localité & $\begin{array}{c}R \\
(\%)\end{array}$ & $\begin{array}{l}\text { 니 } \\
(\%)\end{array}$ & $\begin{array}{c}\text { DL50 TBZ } \\
(\mu \mathrm{g} / \mathrm{ml})\end{array}$ \\
\hline \multicolumn{7}{|l|}{ G ambie } \\
\hline - Ovins & 3 & $\begin{array}{l}1 \mathrm{R} \\
1 \mathrm{R}\end{array}$ & $\begin{array}{l}\text { Bansang } \\
\text { Bansang }\end{array}$ & $\begin{array}{c}84,3^{*} \\
88,6 * *\end{array}$ & $\begin{array}{l}33,1 \\
50,6\end{array}$ & $\begin{array}{c}<0,05 \\
\text { NT }\end{array}$ \\
\hline $\begin{array}{l}\text { - Caprins } \\
\text { Sénégal }\end{array}$ & 1 & 1 SU SP & Banjul & 95,2 & 56,1 & NT \\
\hline - O vins & 15 & $\begin{array}{l}1 \text { SU SP } \\
1 \text { SU SP } \\
1 \text { SU SP }\end{array}$ & $\begin{array}{l}\text { Kolda } \\
\text { Kolda } \\
\text { Niayes }\end{array}$ & $\begin{array}{c}94,8 \\
\text { NT } \\
97,2\end{array}$ & $\begin{array}{c}73,7 \\
\text { NT } \\
72,3\end{array}$ & $\begin{array}{c}0,09 \\
0,1 \\
\text { NT }\end{array}$ \\
\hline - Caprins & 2 & 0 & Niayes & $>95$ & $>90$ & NT \\
\hline
\end{tabular}




\section{Remerciements}

Nous remercions les docteurs B. Goossens et P. Ankers, à S. Kora, le personnel technique de l'International Trypanotolerance Centre (ITC, Banjul), ainsi que l'UBP/Project, le personnel du Centre de recherche zootechnique de Kolda et le Professeur Pangui de l'Eismv de Dakar.

\section{BIBLIO GRAPHIE}

1. BAUER V.C., FAILING K., 1992. Einsath von Anthelmintika zur nematodenbekämpfung bei Schafen in Westdeutschland : Ergebniss und U mfrag. Dtsch. Tierärtzl. W schr., 99: 365-370.

2. BOERSEMA J.H., PANDEY V.S., 1997. Anthelmintic resistance of trichostrongylids in sheep in the highveld of Zimbabwe. Vet. Parasitol., 68: 383-388.

3. BOGAN J., BENOIT E., DELATOUR P., 1987. Pharmacokinetics of oxfendazole in goats: a comparison with sheep. J. vet. Pharmacol. Ther. 10: 305-309.

4. BORGSTEEDE F.H.M., PEKELDER J.J., DERCKSEN D.P., SOL L., VELLEMA P., GAASENBEEK C.P.H., VANDERLINDEN J.N., 1997 A survey of anthelmintic resistance in nematodes of sheep in The N etherlands. Vet. Q uart., 19: 167-172.

5. COLES G.C., BAUER C., BORGSTEEDE F.H.M., GEERTS S., KLEI T.R., TAYLOR M.A., WALLER P.J., 1992. World Association for the Advancement of Veterinary Parasitology (WAAVP) methods for the detection of anthelmintic resistance in nematodes of veterinary importance. Vet. Parasitol., 44: 35-44.

6. COLES G.C., BO RGSTEEDE F.H.M., GEERTS S., 1994. Anthelminticresistance nematodes in the E.U. Parasitol. Today, 10: 288-289.

\section{Summary}

Bâ H., G eerts S. Benzimidazole resistance of gastrointestinal nematodes in small ruminants in The Gambia and Senegal

A survey on benzimidazole $(B Z)$ resistance in gastrointestinal nematodes has been carried out in 55 flocks of sheep and goats in The Gambia and Senegal using the fecal egg count reduction test and egg hatch assay. BZ resistance was suspected in four cases out of 34 examined village flocks. In the 21 improved flocks, however, BZ resistance was present in two cases and suspected in four. This is the first report of BZ resistance in gastrointestinal nematodes of small ruminants in The Gambia and Senegal. Further research should be carried out on a larger scale in order to measure the prevalence of nematodes' anthelmintic resistance in the region and to identify the helminth species involved.

Key words: Sheep - Goat - Nematoda - Benzimidazole Resistance to chemicals - Anthelmintics - The Gambia Senegal.
7. DORNY P., CLAEREBO UT E., VERCRUYSSE J., SANIR R., JALILA A., 1994. Anthelmintic resistance in goats in peninsular Malaysia. Vet. Parasitol., 55: 327-342.

8. FRITSCHE T., KAUFMANN J., PFISTER K., 1993. Parasite spectrum and seasonal epidemiology of gastro-intestinal nematodes of small ruminants in The Gambia. Vet. Parasitol., 49: 271-283.

9. GEERTS S., DORNY P., 1995. Anthelmintic resistance in helminths of animal and man in the tropics. Bull. Séances Acad. R. Sci. O utre-Mer, 41: $401-424$

10. HUNT K.R., TAYLOR A.A., 1989. Use of the egg hatch assay on sheep faecal samples for the detection of benzimidazole resistant nematodes. Vet. Rec., 125: 153-154.

11. LE JAMBRE L.F., 1976. Egg hatch as in vitro assay of thiabendazole resistance in nematodes. Vet. Parasitol., 2: 385-391.

12. NDAO M., BELOT J., ZINSSTAG J., PFISTER K., 1995. Epidémiologie des helminthoses gastro-intestinales des petits ruminants dans la zone sylvo-pastorale au Sénégal. Vet. Res., 26 : 132-139.

13. SANGSTER N.C., RIOCKARD J.M., HEN NESSY D.R., STEEL J.W., COLLINS G.H., 1991. Disposition of oxfendazole in goats and efficacy compared with sheep. Res. vet. Sci., 51: 258-263.

14. VAN WYK J.A., MALAN F.S., RANDLES J.L., 1997. How long before resistance makes it impossible to control some field strains of Haemonchus contortus in South Africa with any of the modern anthelmintics. Vet. Parasitol., 70: 111-122.

15. WALLER P.J., ECHEVARRIAZ E.C., MACIEL S., NARI A., HANSEN J.W., 1996. The prevalence of anthelmintic resistance in nematode parasites of sheep in southern Latin America: General overview. Vet. Parasitol., 62: 181-187.

Reçu le 4.8.97, accepté le 5.11.98

\section{Resumen}

Bâ H., Geerts S. Resistencia a los benzoamidazoles en los nematodos gastrointestinales de los pequeños rumiantes en Gambia y Senegal

Con el fin de detectar una eventual resistencia a los benzoamidazoles, se examinaron 55 hatos de pequeños rumiantes en Gambia y Senegal, mediante el uso del test de inhibición de la eclosión de los huevos y/o el test de reducción de huevos de nematodos en las heces. De los 34 hatos de pueblo examinados, se identificaron quatro casos sospechosos de resistencia a los benzoamidazoles. En los 21 hatos mejorados, por el contrario, se constataron dos casos de resistencia y quatro casos de sospecha de resistencia. El presente estudio permitió demostrar, por primera vez, casos de resistencia a los benzoamidazoles en los nematodos gastrointestinales de los pequeños rumiantes en Senegal y en Gambia. Las investigaciones deberían realizarse a mayor escala, con el fin de medir la prevalencia de la resistencia de los nematodos a los antihelmínticos en la región y de determinar las especies de parásitos involucradas.

Palabras clave: O vino - Caprino - Nematoda - Benzimidazole Resistencia a productos químicos - Antihelmíntico - Gambia Senegal. 